\title{
Développement d'un comparateur cryogénique de courant (CCC) pour la métrologie des faibles courants
}

\author{
Florentin Rengnez ${ }^{1}$, Olivier Séron ${ }^{1}$, Laurent Devoille ${ }^{1}$, François Piquemal $^{1}$ et Dominique Placko ${ }^{2}$ \\ ${ }^{1}$ LNE, 29 avenue Roger Hennequin, 78197 Trappes Cedex, France \\ ${ }^{2}$ ENS Cachan, 61 Avenue du Président Wilson, 94230 Cachan Cedex, France
}

\begin{abstract}
Résumé. Un nouveau comparateur cryogénique de courant (CCC) a été développé au LNE. Avec un gain maximum de 30000 , il sera utilisé pour amplifier et mesurer les faibles courants générés par des pompes à électron actuellement à l'étude pour servir comme étalon quantique de courant. Cet article présente la conception et la réalisation du comparateur et les performances atteintes. Une résolution record très proche de $1 \mathrm{fA} / \mathrm{Hz} 1 / 2$ en régime de bruit blanc a été obtenue. Grâce à une géométrie inédite adoptée pour les enroulements, les phénomènes de résonance émanant des circuits LC par couplage de leur inductance aux capacités parasites existantes sont observés à des fréquences au delà de $1 \mathrm{kHz}$, n'affectant pas ainsi la bande passante du système. Un modèle électrique représentatif du comportement fréquentiel du CCC est en cours de développement.
\end{abstract}

\section{Introduction}

Depuis le $1^{\text {er }}$ janvier 1990, les unités électriques ohm et volt sont reproduites dans le système international d'unités (SI) par la mise en œuvre de l'effet Hall quantique (EHQ) et de l'effet Josephson (EJ). Ces deux phénomènes quantiques ont bouleversé la métrologie électrique en donnant la possibilité de relier directement résistance et tension à deux constantes fondamentales, respectivement la constante de von Klitzing $R_{\mathrm{K}}=h / e^{2}$ et la constante de Josephson $K_{\mathrm{J}}=2 e / h, h$ étant la constante de Planck et $e$ la charge élémentaire. Ces phénomènes permettent ainsi de disposer, sous certaines conditions, d'excellents étalons fondamentaux dont la valeur est immuable dans l'espace et dans le temps et procurent ainsi une représentation unique de l'ohm et du volt au niveau international [1-3].

Bénéficiant de l'ancrage quantique de ces deux unités et des faibles incertitudes afférant à leur réalisation pratique, la reproduction de l'ampère, et la détermination de ses multiples et sous-multiples passent naturellement par les étalons de résistance et de force électromotrice en appliquant la loi d'Ohm. Toute intensité comprise entre 1 $\mathrm{nA}$ et $10 \mathrm{kA}$ est ainsi raccordée à l'ohm et au volt par des mesures de la tension aux bornes d'une résistance et/ou par le biais de comparateur de courant fonctionnant à température ambiante. Pour les intensités de courant inférieures à $1 \mathrm{nA}$, la méthode de mesure repose sur l'utilisation d'un amplificateur intégrateur: par exemple, il s'agit de mesurer sur une certaine durée la variation de tension aux bornes d'un condensateur de capacité connue lorsque celui-ci est chargé par le courant à mesurer.

La figure 1 représente les meilleures incertitudes CMC [4] correspondant à la grandeur " intensité de courant continu » publiées dans la banque de données du BIPM accessible sur internet [5]. Elles n'ont été obtenues que par les méthodes traditionnelles mentionnées précédemment et sans faire intervenir directement la mise en œuvre de l'EHQ et de l'EJ. L'incertitude la plus faible, légèrement inférieure à $10^{-6}$ en valeur relative, est obtenue pour des intensités de $1 \mu \mathrm{A}$ à $10 \mathrm{~mA}$ en courant continu. Cependant, l'incertitude pourrait être réduite d'un facteur 10 à 100 en impliquant directement les étalons quantiques de tension et de résistance et l'utilisation d'un comparateur cryogénique de courants (CCC), cela dans une gamme de courant légèrement plus étendue mais n'allant pas en deçà de $1 \mathrm{nA}$. Dans la figure 6 , l'ensemble des valeurs correspondant à EHQ/EJ+CCC provient de la combinaison des meilleures valeurs CMC de résistance et de tension. Les valeurs SET et SET $+\mathrm{CCC}$ sont calculées à partir des performances en bruit attendues des dispositifs concernés. 
Figure 1. Incertitude-type déduite des valeurs $\mathrm{CMC}$ pour différentes méthodes d'étalonnage de courant.

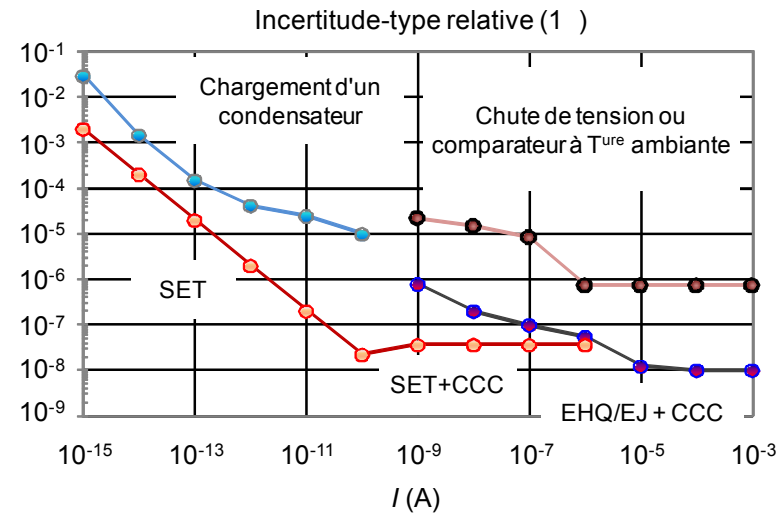

Enfin, une comparaison internationale récente [6] de très faibles intensités, comprises entre 100 fA et $100 \mathrm{pA}$, confirme l'ordre de grandeur des CMCs dans cette gamme. Elle montre un bon accord entre les participants avec une incertitude relative élargie $(k=2)$ des valeurs de référence comprises entre $2,7 \cdot 10^{-4}$ et $1,9 \cdot 10^{-5}$.

La réalisation d'un étalon quantique de courant utilisant l'effet tunnel à un électron (en anglais SET : Single electron tunnelling) offre de nouvelles perspectives dans la mesure des faibles courants en allant au-delà des possibilités d'étalonnage que pourraient offrir l'EHQ et l'EJ.

Dans certains dispositifs SET, communément appelés pompe à électron, il est possible de contrôler le courant, électron par électron, en combinant la répulsion coulombienne entre électrons et leur passage par effet tunnel à travers une barrière isolante. Lorsque ces transferts d'électrons sont cadencés par un signal RF de fréquence $f$, le courant $I$ traversant le circuit sera alors donné par la relation : $I=e \cdot f$. En mesurant la fréquence, on dispose d'un étalon de courant dont la valeur est ici universelle, simplement reliée au quantum de charge $e$ [7]. Les pompes à électron actuellement à l'étude ont des fréquences de fonctionnement allant de $10 \mathrm{MHz}$ à $1 \mathrm{GHz}$ conduisant ainsi à des intensités de courant de $1,6 \mathrm{pA}$ à $160 \mathrm{pA}[8,9]$.

La figure 1 montre que l'association d'une pompe à électron et d'un CCC utilisé comme amplificateur de courant, avec l'hypothèse que ce dernier dispose d'une résolution de $1 \mathrm{fA} / \mathrm{Hz}^{1 / 2}$ à basse fréquence $(1 \mathrm{~Hz})$ en régime de bruit blanc, permettrait d'obtenir une incertitude relative environ 100 fois meilleure sur la gamme de courant 100 pA $100 \mathrm{nA}$ par rapport aux méthodes traditionnelles. Pour des intensités plus faibles, la pompe à électron seule conduirait à une réduction d'incertitude dans un facteur 10.

Un tel étalon quantique fondé sur un dispositif SET et associé à un CCC utilisé comme amplificateur de courant mais aussi au préalable comme instrument de mesure pour la caractérisation métrologique de ces dispositifs monoélectroniques, devrait donc améliorer de façon significative la chaîne de traçabilité des faibles intensités de courant. Cet axe de progrès que le LNE s'est engagé à suivre à l'instar d'autres laboratoires nationaux de métrologie, particulièrement européens [9] bénéficiera clairement :

(i) aux laboratoires nationaux eux-mêmes pour leurs activités d'étalonnage sur les sub-nano ampèremètres, les étalons de courant secondaires ou les étalons de résistance de très hautes valeurs $(R>1 \mathrm{~T} \Omega)$ ou pour leurs activités impliquant des moyens de référence de haut niveau pour la mesure des faibles valeurs de courant ou de charge, notamment dans le domaine des rayonnements ionisants;

(ii) aux fabricants d'instruments de mesure (détecteurs ou mesureurs de faibles signaux électriques) ;

(iii) à l'industrie de la micro et nanoélectronique pour la caractérisation des dispositifs semiconducteurs, des micro ou nano systèmes électromécaniques (MEMS/NEMS) ou encore des dispositifs nanostructurés ou nanomatériaux, des films minces conducteurs, des matériaux organiques ou des nanotubes de carbone [10] (caractéristiques courant-tension, tests électriques de " wafers », mesures de courant de fuite ou de spectre de bruit en courant, mesures de conductance ou d'impédance sous faible niveau de signal d'excitation ...).

La pertinence du développement d'un tel étalon quantique de courant est encore plus claire dans le cadre d'une redéfinition de l'ampère et la mise en pratique correspondante, qui devrait être formulée ainsi [11]:

"L'ampère A est l'unité de courant électrique ; son amplitude est définie en fixant la valeur numérique de la charge élémentaire, égale exactement à 1,602 17X quand elle est exprimée en A.s, unité égale au coulomb», le symbole $\mathrm{X}$ correspondant à un ou plusieurs chiffres selon la valeur de $e$ résultant de l'ajustement le plus récent de CODATA une fois les incohérences levées [12]. Cette définition sera accompagnée d'un texte explicatif dans la brochure du SI :

«Ainsi, nous avons la relation exacte $e=1,60217 \mathrm{X} \cdot 10^{-19} \mathrm{C}$. L'effet de cette définition est que l'ampère est le courant électrique correspondant au débit de $1 /\left(1,60217 \mathrm{X} \cdot 10^{-19}\right)$ charges élémentaires par seconde ».

Cela diffère de façon drastique avec la définition actuelle: " L'ampère est l'intensité d'un courant constant qui, maintenu dans deux conducteurs parallèles, rectilignes, de longueur infinie, de section circulaire négligeable et placés à une distance de 1 mètre l'un de l'autre dans le vide, produirait entre ces conducteurs une force égale à $2 \cdot 10^{-7}$ newton par mètre de longueur. »

Dans cet article, nous rapportons les derniers résultats sur le développement d'un $\mathrm{CCC}$ de gain 30000 destiné à terme à l'étude des pompes à électron à barrière ajustable à base de nanofils en silicium développées par le CEA-INAC avec le CEA-LETI [8]. Les principaux résultats présentés ici sont issus des mesures de spectre de bruit et des travaux de modélisation. Au préalable, nous rappelons le principe du 
CCC et donnons des éléments de conception et de sa réalisation.

\section{Principe du CCC}

Inventé par Harvey en 1972 [13], l'utilisation du CCC est très répandue en métrologie électrique, d'une part au sein de pont de résistances (c'est le CCC qui a permis d'établir la très grande reproductibilité des étalons quantiques de résistance), et pour l'amplification et la mesure des faibles courants délivrés par des pompes à électron d'autre part [14].

Le principe du CCC repose sur la loi d'Ampère et le diamagnétisme parfait d'un supraconducteur dans l'état Meissner. Considérons deux fils insérés dans un tube supraconducteur de longueur infinie et d'épaisseur plusieurs fois supérieure à la longueur de pénétration de London (fig. 2).

Figure 2. Principe du CCC

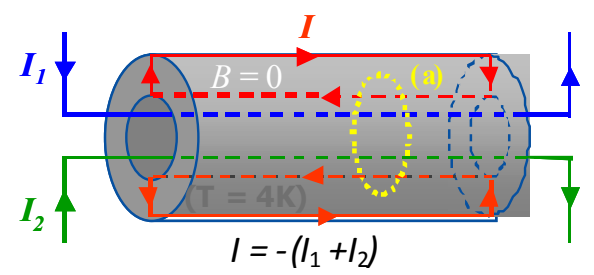

Les courants $I_{1}$ et $I_{2}$ traversant ces fils induisent un supercourant $I$ circulant de l'intérieur du tube vers l'extérieur de manière à annuler la densité de flux magnétique dans le tube. En appliquant la loi d'Ampère à un contour dans l'épaisseur du tube, noté $a$, nous obtenons :

$$
\oint_{a} B d l=0=\mu_{0}\left(I_{1}+I_{2}-I\right)
$$

d'où l'égalité des courants : $I=I_{1}+I_{2}$.

Si les fils transportant les courants $I_{1}$ et $I_{2}$ passent dans le tube respectivement $N_{1}$ et $N_{2}$ fois, le supercourant sera alors égal à :

$$
I=N_{1} I_{1}+N_{2} I_{2}
$$

Ces égalités sont valables indépendamment de la position des fils dans le tube. Seule la distribution de densité de courant sur la surface interne du tube peut être inhomogène dans le cas d'un positionnement asymétrique des fils, alors que la distribution de densité de courant sur la surface externe du tube est homogène. C'est la raison clé du haut niveau d'exactitude du rapport de courants d'un CCC.

Dans le cas réel d'un tube de longueur finie, les égalités cidessus sont altérées par le champ magnétique non écranté induit par les courants $I_{1}$ et $I_{2}$ aux extrémités. Pour surmonter ces effets d'extrémités et en même temps avoir un dispositif pratique de taille raisonnable, le tube est façonné en tore, ses extrémités se recouvrant tout en étant électriquement isolées (fig. 3). Plus la longueur de recouvrement est grande, plus les fuites de flux magnétique sont faibles.

Les courants $I_{1}$ et $I_{2}$ de direction opposée circulant dans deux enroulements de nombre de tours $N_{1}$ et $N_{2}$ induisent un supercourant $I=N_{1} I_{1}-N_{2} I_{2}$. Le flux magnétique externe $\Phi$, seule conséquence du supercourant, est détecté par un SQUID (Superconducting Quantum Interference Device) à travers un transformateur de flux composé d'une bobine de détection positionnée au plus près du blindage toroïdal et connectée à l'inductance d'entrée du SQUID. La tension de sortie du détecteur de flux est ensuite convertie en courant qui sera réinjecté dans un des deux enroulements pour annuler les forces magnétomotrices dans le CCC. De cet équilibre en ampère tour résulte l'égalité des rapports: $I_{2} / I_{1}=N_{1} / N_{2}$.

Figure 3. Schéma d'un CCC

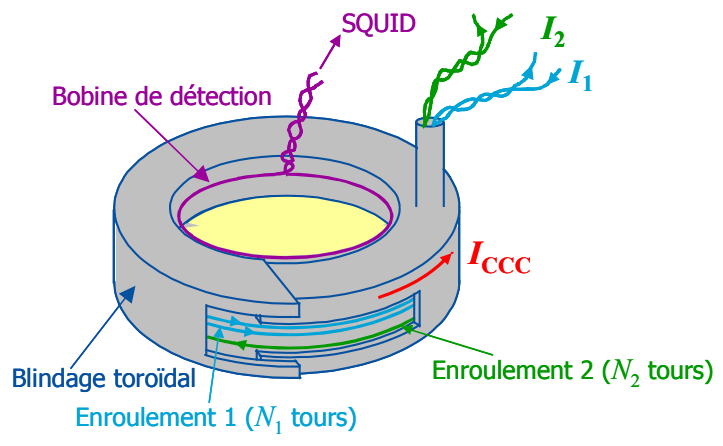

\section{Conception et fabrication}

Le CCC est composé de trois enroulements de 10000 tours, deux enroulements de 1 tour, un enroulement de 100 tours et un enroulement de 99 tours. Les enroulements sont réalisés à partir de fil en $\mathrm{NbTi}$ de $65 \mu \mathrm{m}$ de diamètre dans une matrice en cuivre et fixés dans de l'époxy. Le blindage toroïdal recouvrant les enroulements est réalisé avec des feuilles de plomb de $100 \mu \mathrm{m}$ d'épaisseur. Chaque recouvrement est isolé des autres en utilisant du scotch en polytétrafluoroéthylène (PTFE). Le tore a un diamètre intérieur de 19,2 $\mathrm{mm}$, un diamètre extérieur de 52,8 $\mathrm{mm}$ pour une hauteur de 23,4 $\mathrm{mm}$. Ces dimensions respectent les contraintes d'encombrement liées au cryostat.

Le CCC a été couplé à un SQUID fabriqué par Magnicon et sélectionné pour sa très bonne résolution en énergie $\left(\varepsilon_{\mathrm{SQ}}=\right.$ $212 h$ à $1 \mathrm{~Hz}$ ). Le système global \{CCC-transformateur de flux-SQUID $\}$ montré dans la figure 4 est enfermé dans quatre blindages cylindriques coaxiaux. Le blindage extérieur est composé de matériau ferromagnétique permettant un premier écrantage du champ magnétique environnemental, de sorte que les trois autres blindages en plomb trouvent leur pleine efficacité à atténuer le champ magnétique résiduel et à le stabiliser au niveau du CCC. 


\section{Web of Conferences}

Figure 4. Image du CCC de gain 30000 et du SQUID.

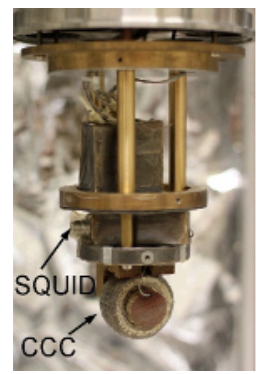

Sur la base des travaux antérieurs publiés dans la littérature (voir références dans [3]), le fonctionnement du CCC peut être affecté dramatiquement par des phénomènes de résonance émanant principalement des circuits oscillants que constituent les plus grands enroulements avec les capacités parasites existantes au sein du CCC. Elles peuvent être excitées par différentes sources: le signal de modulation du SQUID transmis par le transformateur de flux; le signal de déséquilibre dynamique des enroulements lors des rampes de courant, ou lors des inversions de polarité du courant; des signaux parasites circulant dans les circuits primaire et secondaire du système. A des fréquences trop basses, les résonances peuvent aussi induire une élévation importante du plancher de bruit, en étant stimulées par des sources de vibration acoustique ou mécanique. Plusieurs pistes ont été proposées pour minimiser l'impact de la résonance interne sur le fonctionnement propre du CCC. Dans ce même but, alors que les CCC ont usuellement des enroulements qui se superposent radialement couche par couche, nous avons opté pour un agencement des enroulements du CCC par empilement axial (fig.5) afin de réduire les capacités parasites entre enroulements et donc observer une augmentation significative de la valeur des fréquences de résonance, $f_{\mathrm{R}}$ variant en $1 /\left[2 \pi(L C)^{1 / 2}\right]$ où $L$ est l'inductance de l'enroulement considéré.

Figure 5. a) Superposition radiale conventionnelle des enroulements; b) superposition axiale des enroulements. a)

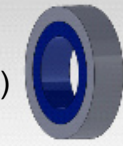

b)

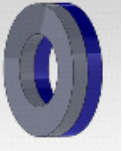

\section{Résultats}

\subsection{Sensibilité et résolution en courant}

La sensibilité $S_{\mathrm{CCC}}$, définie par le supercourant $I$ circulant sur la surface du tube de recouvrement du CCC induisant une variation d'un quantum de flux $\Phi_{0}$ au niveau du SQUID, a été trouvée égale à $S_{\mathrm{CCC}}=8,8 \mu \mathrm{A} . \mathrm{t} / \Phi_{0}$. Elle s'écarte de la valeur optimale de $6 \mu \mathrm{A} . t / \Phi_{0}$ donné par la relation $S_{\mathrm{CCC}}=2 \cdot N^{\mathrm{opt}} \cdot S_{\mathrm{SQUID}}$ ou $N^{\mathrm{opt}}$ est le nombre de tours optimal de la bobine de détection $\left(N^{\text {opt }}=6\right)$ et la sensibilité du SQUID utilisé $S_{\text {SQUID }}=0,5 \mu \mathrm{A} / \Phi_{0}$. Les optimisations du comparateur conduisent à un niveau de bruit relativement proche du niveau attendu, avec une valeur en termes de flux magnétique de $3,7 \mu \Phi_{0} / \mathrm{Hz}^{1 / 2}$ à $1 \mathrm{~Hz}$ à comparer à la contribution en bruit de $2,8 \mu \Phi_{0} / \mathrm{Hz}^{1 / 2}$ qu'apporte le SQUID (fig. 6). Cela conduirait à une résolution en courant de valeur inégalée de $1,5 \mathrm{fA} / \mathrm{Hz}^{1 / 2}$ à $1 \mathrm{~Hz}$ (la valeur théorique étant de $0,8 \mathrm{fA} / \mathrm{Hz}^{1 / 2}$ à $1 \mathrm{~Hz}$ pour 30000 tours).

Il est à noter une valeur un peu plus élevée de la fréquence de recouvrement, $f_{\mathrm{C}} \approx 2 \mathrm{~Hz}$ délimitant le bruit en $1 / \mathrm{f}$ du bruit blanc dont le plancher se situe à une valeur de $3 \mu \Phi_{0} / \mathrm{Hz}^{1 / 2}$ sur un domaine de fréquence particulièrement étendu $\{2 \mathrm{~Hz}$ $-1 \mathrm{kHz}\}$. Sur la plage de fréquence $\{2 \mathrm{~Hz}-6 \mathrm{~Hz}\}$ adaptée aux mesures de courant, nous disposerions d'une résolution du CCC de l'ordre de $1,1 \mathrm{fA} / \mathrm{Hz}^{1 / 2}$ qui est une valeur record.

Figure 6. Spectre de bruit en flux magnétique du CCC. Les lignes en trait tiré indiquent le plancher de bruit blanc au niveau de $3 \mu \Phi_{0} / \mathrm{Hz}^{1 / 2}$ et le bruit en $1 / \mathrm{f}$ (en dessous de $2 \mathrm{~Hz}$ ). Le pic à $6 \mathrm{kHz}$ traduit la résonance selfique $(L C)$ du CCC.

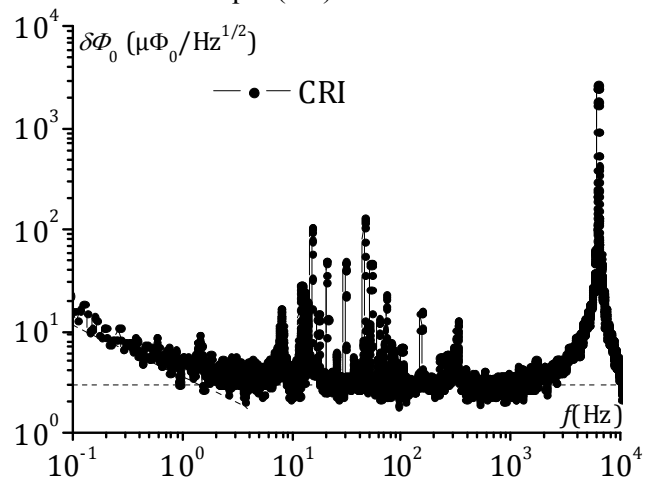

Le bruit en flux a été mesuré avec le SQUID en mode de contre-réaction interne (CRI), qui est le mode de verrouillage en flux par défaut du SQUID utilisé. Le courant de contre-réaction alimente une bobine directement couplée au SQUID. Ce mode, par construction plus simple à mettre en œuvre, plus stable avec une large bande passante s'avère particulièrement bien adapté pour les premières phases de caractérisation des dispositifs à un électron mais le gain du système global d'amplification est imprécis.

Nous avons également testé le mode fondé sur une contre réaction externe qui est le mode de fonctionnement usuel du CCC. Le SQUID est verrouillé en flux en délivrant un courant dans l'enroulement secondaire du CCC $\left(N_{2}\right.$ tours $)$ au moyen d'une source dont l'intensité de courant est régulée par le courant de contre-réaction du SQUID. Cependant, l'électronique utilisée dans ce dernier mode nécessite des améliorations de l'électronique. Plusieurs pistes sont envisagées: réduire les courants de fuites à la masse par isolation optique, améliorer les filtres contre les perturbations extérieures (50 Hz et les harmoniques)... 


\subsection{Simulation}

Les enroulements du CCC, se comportant comme des circuits résonants $L C$ par couplage de leur inductance avec les capacités parasites, vont induire des fréquences de résonance susceptibles de perturber le bon fonctionnement du système (voir le pic à $6 \mathrm{kHz}$ sur la figure 6). A l'instar des imperfections du blindage toroïdal de recouvrement, les résonances peuvent aussi se traduire par des erreurs de gain significatives [3]. Leur appréhension est donc indispensable pour tout développement de CCC et cela passe par la détermination d'un modèle électrique équivalent représentatif du comportement fréquentiel du CCC.

Nous avons étudié les trois principales configurations du CCC qui permettent d'obtenir des rapports de courants de 10000,20000 et 30000 . Plusieurs pics de résonance ont été observés sur le spectre de bruit en flux magnétique mesuré à la sortie du SQUID respectivement en laissant les trois enroulements de 10000 tours déconnectés (cas de la figure 6) ou en mettant en série deux des enroulements ou les trois ensemble. Pour chaque configuration, un circuit électrique équivalent du CCC a été réalisé, via le logiciel Pspice. Cette modélisation a nécessité d'effectuer des mesures de plus de dix paramètres (inductances, capacités parasites, résistances) au moyen d'un $R L C$ mètre QuadTech de type 7400 . Le tableau 1 récapitule les valeurs de fréquence de résonance obtenues par la simulation à comparer avec les valeurs obtenues à partir des spectres de bruit.

Tableau 1. Fréquences de résonance par mesure et simulation.

\begin{tabular}{|c|c|c|c|c|}
\hline \multirow{2}{*}{$f_{\mathrm{R}}(\mathrm{kHz})$} & \multicolumn{2}{|c|}{ Mesure } & \multicolumn{2}{|c|}{ Simulation } \\
\hline & \multicolumn{2}{|c|}{$1^{\mathrm{er}} \mathrm{pic} \quad 2^{\mathrm{ème}} \mathrm{pic}$} & \multicolumn{2}{|c|}{$1^{\mathrm{er}}$ pic $\quad 2^{\text {ème }}$ pic } \\
\hline $10^{4}$ tours & 6 & 14,6 & 6,1 & 17 \\
\hline $2 \times 10^{4}$ tours & 4,8 & 13,1 & 4,7 & 12,6 \\
\hline \multirow{2}{*}{$3 \times 10^{4}$ tours } & \multirow{2}{*}{3,2} & 11,5 & \multirow{2}{*}{3,1} & 13,8 \\
\hline & & 12,8 & & 15,3 \\
\hline
\end{tabular}

Nous retrouvons par la simulation les deux pics de résonnance observés sur les spectres de bruit pour les trois configurations de mesure et le dédoublement du $2^{\text {ème }}$ pic dans le cas des trois enroulements connectés. Pour les trois configurations, les valeurs calculées et mesurées des fréquences de résonance du $1^{\mathrm{er}}$ pic sont très proches. En revanche, il existe un écart significatif des positions des deuxièmes pics. L'amélioration de la modélisation du CCC en circuit électrique équivalent pour les trois configurations données nécessite de comparer les spectres d'impédance mesurées directement à partir du $R L C$ mètre et les spectres d'impédance obtenues par simulation, cela sur une gamme de fréquence la plus large possible (typiquement entre $10 \mathrm{~Hz}$ et $500 \mathrm{kHz}$ ).

L'expression analytique de grandeurs électriques relatives au circuit considéré comme équivalent aux enroulements (impédance, erreur de rapport de courants en courant alternatif ...) est nécessaire pour une caractérisation métrologique complète du CCC. Dans cette optique, une nouvelle méthode dite matricielle a été développée. Elle consiste à considérer un schéma électrique, à écrire les équations provenant de la loi des mailles et des nœuds, puis à les regrouper en une seule équation :

$$
K=[M] I
$$

composée d'un vecteur $K$, contenant les conditions aux limites, dépendant d'un vecteur $I$ contenant tous les courants à déterminer, et d'une matrice $M$ dite "d'intéraction ", regroupant tous les paramètres du problème, i.e. : inductances, mutuelles inductances, capacités, résistances et la fréquence. L'inversion de la matrice permet d'obtenir les solutions $\mathrm{du}$ problème et d'en déduire l'expression analytique de toutes les grandeurs du circuit: tensions, courants, impédances, phases etc.

Une nouvelle campagne de mesures d'inductances et de mutuelles a permis de comprendre le comportement en basse fréquence $(<100 \mathrm{~Hz})$ du système quelle que soit la configuration utilisée: enroulements court-circuités ou en série par exemple. Le modèle utilisé pour la suite comprend les inductances des enroulements, les coefficients de couplage, la capacité parasite aux bornes de chaque enroulement et la résistance des fils. La figure 7 montre le bon accord entre la mesure d'impédance (courbe noire) et la simulation (courbe bleue) dans le cas d'un enroulement de 10000 tours, les autres enroulements étant déconnectés.

Figure 7. Comparaison entre une mesure d'impédance via un RLC mètre (courbe noire) et le modèle (courbe bleue) pour un enroulement de 10000 tours.

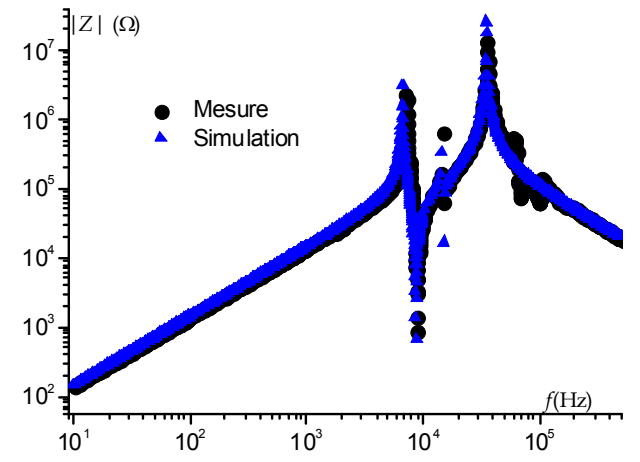

Nous avons ensuite testé les limites de validité du modèle dans les configurations plus complexes où les deux puis les trois enroulements de 10000 tours sont en série. La figure 8 regroupe les résultats pour 30000 tours.

Figure 8. Comparaison entre une mesure d'impédance (courbe noire) et la simulation (courbe bleue) avec tous les enroulements de 10000 tours en série. 


\section{Web of Conferences}

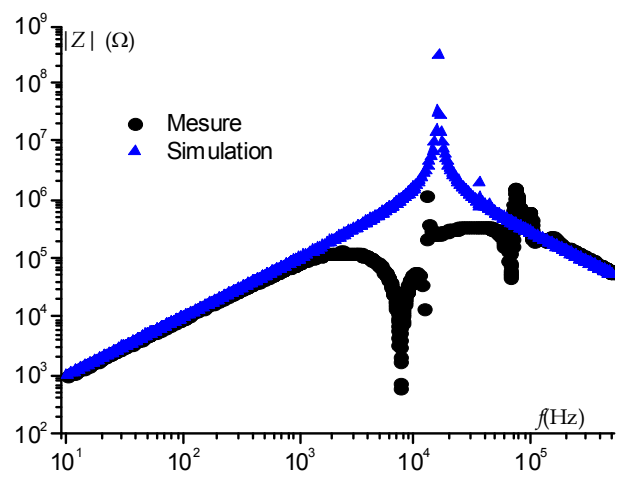

Dans ces cas, le modèle est représentatif du comportement $\mathrm{du}$ système pour des fréquences inférieures à $1 \mathrm{kHz}$ et supérieures à $100 \mathrm{kHz}$, mais il existe un désaccord dans la gamme intermédiaire.

Un nouveau modèle est en cours d'étude en intégrant les capacités parasites existantes entre les enroulements et le blindage toroïdal, les capacités des fils allant de l'extérieur du système $(296 \mathrm{~K})$ au CCC situé en bas du cryostat (4,2 K), les capacités à la masse, etc.

\subsection{Exactitude de rapport de courant}

Une des caractéristiques essentielles du CCC est l'exactitude du rapport des courants continus $I_{2} / I_{1}$, qui théoriquement est égal au rapport du nombre de tours des enroulements $N_{1} / N_{2}$ lorsque le supercourant $I$ est nul. L'objectif ultime d'incertitude sur le rapport des nombres de tours $N_{1} / N_{2}$ est $10^{-8}$. Pour mesurer son exactitude, deux enroulements de même nombre de tours $N$ sont connectés en série-opposition et une rampe de courant est appliquée de $0 \mu \mathrm{A}$ à $10 \mu \mathrm{A}$ pour $N=10^{4}$ et jusqu'à $12 \mathrm{~mA}$ lorsque $N=1$, cela en inversant la polarité une fois, à chaque pas. Le tableau 2 regroupe les écarts obtenus.

Tableau 2. Ecarts mesurés $\left(\varepsilon_{\mathrm{dc}}\right)$ et incertitude de type A $(u)$ pour les enroulements de 1 tour et 10000 tours.

\begin{tabular}{|c|c|}
\hline Configuration & $\begin{array}{c}3 \text { recouvrements du } \\
\text { blindage toroïdal } \\
\left(\varepsilon_{\mathrm{dc}} \pm u\right) \times 10^{-6}\end{array}$ \\
\hline $1 \mathrm{t}: 1 \mathrm{t}$ & $=>\mathrm{qq} 10^{-7}$ \\
\hline $10^{4} \mathrm{t}(1): 10^{4} \mathrm{t}(2)$ & $0,0004 \pm 0,0002$ \\
\hline $10^{4} \mathrm{t}(1): 10^{4} \mathrm{t}(3)$ & $0,0004 \pm 0,0003$ \\
\hline $10^{4} \mathrm{t}(2): 10^{4} \mathrm{t}(3)$ & $0,0003 \pm 0,0002$ \\
\hline
\end{tabular}

Concernant les enroulements de 10000 tours, l'exactitude est de quelques $10^{-10}$. En revanche, pour les enroulements de 1 tour, l'ordre de grandeur est de quelques $10^{-7}$ au mieux. Les erreurs obtenues avec les enroulements de 1 tour sont en cours d'investigation. Elles pourraient provenir de la sortie axiale des fils des enroulements par rapport au blindage toroïdal. Des pistes d'amélioration sont envisagées.

\section{Conclusion}

Un CCC de rapport $30000: 1$ a été développé au LNE dans le cadre du développement d'un étalon quantique de courant. Son niveau de bruit, la méthode de superposition axiale adoptée pour les plus grands enroulements et les valeurs élevées de fréquences de résonance qui en découlent constituent des résultats encourageants qui ouvrent la voie à la construction de CCC de gain plus élevé $(>30000)$ et d'une résolution inférieure à $1 \mathrm{fA} / \mathrm{Hz}^{1 / 2}$. Une nouvelle méthode prometteuse de modélisation du CCC a également été illustrée. De plus amples résultats et analyses de données seront discutés pendant la conférence.

\section{Remerciements}

Ce travail est en partie financé par la Commission Européenne au sein du projet JRP SIB07 Qu-Ampere dans le cadre de l'appel à projet EMRP Call 2011.

\section{Références}

[1] W. Poirier, F. Schopfer, Eur. Phys. J. - Special Topics, vol. $172,207,2009$

[2] B. Jeanneret, S. Benz, Eur. Phys. J. - Special Topics, vol. $172,181,2009$

[3] F. Piquemal, «Quantum Electrical Standards» Handbook of Metrology, édité par Michael Gläser et Manfred Kochsiek, vol. 1 et 2, Wiley-VCH, avril 2010

[4] CMC: Calibration and Measurement Capabilities : aptitudes d'un laboratoire national de métrologie en matière de mesures et d'étalonnages

[5] http://kcdb.bipm.org/

[6] G-D Willenberg, EURAMET.EM-S24 report,

Metrologia, vol. 50, 2013

[7] J. Pekola et al., arXiv:1208.4030, 2013

[8] X. Jehl et al., ce recueil

[9] H. Scherer, ce recueil

[10] R. K. Leach et al., Nanotechnology, vol. 22, 2011

[11] Rapport de la $20^{\text {ème }}$ réunion Comité Consultatif pour les Unités. 14-16 septembre 2010

[12] P. J. Mohr, et al., CODATA Recommended Values of the Fundamental Physical Constants, 2012

[13] I. K. Harvey, Rev. Sci. Instrum., vol. 43, 1972.

[14] J. Gallop and F. Piquemal, SQUIDs Handbook, vol.II, édité par Alex Braginski et John Clark, Wiley, Berlin, 2006. 